\title{
Interações e habilidades sociais entre universitários: um estudo correlacional
}

\author{
Transactions and social skills among university \\ students: a correlation study
}

\author{
Gleiber COUTO' \\ LUC VANDENBERGHE² \\ Wanessa Marques TAVARES ${ }^{3}$
}

Renata Limongi França Coelho SILVA ${ }^{4}$

\begin{abstract}
Resumo
As interações interpessoais são a base de todos os processos sociais. O objetivo deste estudo foi verificar como essas interações se relacionam com habilidades sociais e, ao mesmo tempo, investigar evidências de validade para o Checklist de Relações Interpessoais-II. Participaram 542 estudantes de diversos cursos de ensino superior do sudeste goiano, com idade entre 18 e 55 anos $(M=21, D P=5,48)$, respondendo ao Inventário de Habilidades Sociais, de Del Prette, e ao Checklist de Relações Interpessoais-II. Os participantes com altos escores em habilidades sociais apresentaram mais posições interpessoais do quadrante Amigável-Dominador, enquanto o grupo de baixas habilidades sociais apresentou posições de Hostilidade-Submissão. Além disso, as posições interpessoais do grupo com boas habilidades sociais mostram mais variedade de posições interpessoais. As habilidades sociais têm correlações positivas com posições amigáveis, e correlações negativas com posições hostis e submissas. Esses resultados apoiam a validade para o checklist de relações interpessoais-II.
\end{abstract}

Unitermos: Habilidades sociais. Relações interpessoais. Universitários. Validade de teste.

\begin{abstract}
Interpersonal transactions are the basis of all social processes. The aim of this study was to verify how these transactions are related to social skills, and at the same time, investigate the validity of the Brazilian version of the Checklist for Interpersonal Transactions-II. Participants were 542 students of several university courses in the South Eastern Brazilian State of Goiás; aged between 18 and 55 years ( $M=21, S D=5.48)$. They responded to Social Skills Inventory (Del Prette) and the Checklist for Interpersonal Transactions-II. Participants with the highest scores in social skills were more frequently placed in the Friendly-Dominant quadrant, while the group with the lowest social skills more readily fell into the Hostile-Submissive quadrant. Moreover, the interpersonal positions taken by highly socially skilled students were more varied. Social skills correlate positively with Friendly positions and negatively with Hostile and Submissive positions. These correlations support the validity of the Checklistfor Interpersonal Transactions-II.
\end{abstract}

Uniterms: Social skills. Interpersonal relationships. Collegestudents. Test validity.

\section{$\boldsymbol{\nabla \nabla \nabla \nabla}$}

1 Universidade Federal de Goiás, Departamento de Psicologia, Laboratório de Avaliação, Medidas e Instrumentação em Ciências da Saúde. Av. Dr. Lamartine Pinto de Avelar, 1120, Setor Universitário, 75704-900, Catalão, GO, Brasil. Correspondência para/Correspondence to: G. COUTO. E-mail: <gleibercouto@yahoo.com.br>.

2 Pontifícia Universidade Católica de Goiás, Centro de Ciências Humanas, Programa de Pós-Graduação Stricto-Sensu em Psicologia. Goiânia, GO, Brasil.

3 Universidade Federal de Minas Gerais, Faculdade de Filosofia e Ciências Humanas, Programa de Pós-Graduação em Psicologia. Belo Horizonte, MG, Brasil.

4 Centro de Ensino Superior de Catalão, Curso de Direito. Catalão, GO, Brasil.

Apoio: À Fundação de Amparo à Pesquisa do Estado de Goiás. 
As relações interpessoais são entendidas como associações entre duas ou mais pessoas, que variam segundo o tempo de duração. Elas estão na base da sociedade em geral e são formadas no contexto social sob influências múltiplas, podendo ser reguladas por lei, costume ou acordo mútuo (Berscheid \& Peplau, 1983). Um relacionamento é normalmente visto como uma conexão entre dois indivíduos, por exemplo, um par romântico, um pai e seu filho, ou uma díade de amizade, geralmente envolvendo algum nível de interdependência e transitividade. Por interdependência entendese que pessoas em relação tendem a influenciar-se mutuamente; assim, a maioria das coisas que impactam um membro da relação tem algum nível de impacto sobre o outro membro. Já por transitividade entende-se a expansão da rede de relacionamentos, por exemplo, quando uma pessoa se torna amiga do amigo de um amigo comum (Rusbult \& Buunk, 1993).

O modelo proposto por Levinger (1983) descreve o desenvolvimento natural de um relacionamento em cinco etapas: Conhecimento, Acúmulo, Continuação, Deterioração e Rescisão. Dessa forma, todo relacionamento tem início, meio e fim, sendo que, em relacionamentos de sucesso, o fim se daria pela morte de um dos membros da díade.

Além disso, a causalidade nos processos psicológicos, desde Sullivan (1953), pode ser compreendida a partir de trocas interpessoais e da capacidade destas em atender às necessidades das pessoas envolvidas. As causas de sentimentos e problemas psicológicos não são internas ao indivíduo, mas se constroem a partir da interação. A função das ações interpessoais é evocar, nas pessoas com quem se interage, reações que correspondam às necessidades dos sujeitos que as praticam. Esse processo é recíproco. Se uma díade interage repetidamente, a continuidade de suas trocas leva à formação de certas regularidades, como na dança, onde o movimento de cada parceiro determina os movimentos do outro. $O$ efeito que as reações de cada um têm sobre o outro molda o comportamento do primeiro, e as características de entrosamento entre ambos determinam a qualidade dessa dança.

Dessa maneira, as interações interpessoais podem ser caracterizadas conforme sua capacidade de produzir os efeitos pessoais e sociais desejados, ou seja, 668 nos termos da satisfação das necessidades individuais.
O efeito social que as interações produzem é muitas vezes avaliado como adequado ou não, e as habilidades sociais representam o conjunto de comportamentos que permitem o desempenho social ajustado. Este desempenho é avaliado pela competência social, que se revela pela capacidade de um indivíduo se engajar em situações interpessoais de forma a alcançar os objetivos da interação, mantendo o equilíbrio de poder e as trocas positivas na relação com as outras pessoas.

Tradicionalmente, a competência social é definida como efetividade em interações sociais. Essa definição inclui a capacidade de assumir a perspectiva do outro na interação, tanto quanto a própria perspectiva. Inclui, além das estratégias interpessoais, a capacidade de tomar iniciativas, responder às iniciativas do outro, modificar sua compreensão da situação e do próprio comportamento, dependendo do contexto, das características dos alvos e de sua importância (Z.A.P. Del Prette \&A. Del Prette, 1999;2001a).

A competência social é operacionalizada em diferentes formas na Psicologia, a saber: (1) competência social, avaliada por meio de habilidades sociais estabelecidas; (2) status sociométrico do indivíduo num grupo; (3) quantidade e características dos relacionamentos estabelecidos; e (4) resultados obtidos em tarefas interpessoais (Dirks, Treat \& Robin-Weersing, 2007; Rose-Krasnor, 1997).

Segundo Erickson e Shultz (2001), a principal característica da competência social é a capacidade de monitoramento dos contextos, que permite identificar e mesmo antecipar mudanças. Também está intimamente relacionada com a adaptação e o crescimento pessoal, e ainda com o respeito pelo outro (Z.A.P. Del Prette \& A. Del Prette, 1999). Há uma longa tradição de pesquisa que aponta a competência social como fator de proteção que contribui para a promoção da resiliência pessoal (Garmezy \& Masten, 1994; Luthar, Cicchetti \& Becker, 2000; Cavell, Meehan \& Fiala, 2003; Lillvist, Sandberg, Björck-Äkesson \& Granlund, 2010).

Bellack e Morrison (1982) apontaram o aprendizado constante como necessário para a aquisição e manutenção de um repertório adequado de habilidades sociais, tendo em vista as frequentes mudanças nas contingências sociais. A manutenção de um bom repertório de habilidades sociais apresenta relação direta com a previsibilidade do nível de felicidade que um sujeito 
terá no futuro (Argyle \& Lu, 1990). Além disso, estas habilidades relacionam-se com qualidade de vida e saúde ( Kapp-Simon, MacGuire, Long \& Simon, 2005; Sousa, Galante \& Figueiredo, 2003) e facilitam a satisfação conjugal, sendo enfocadas nas intervenções em crises conjugais (Sardinha, Falcone \& Ferreira, 2009; Stanley, Amato, Johnson \& Markman, 2006). Além disso, elas atuam como fator de proteção contra o tabagismo (Abroms, Simons-Morton, Haynie \& Chen, 2005; Väänänen, Kouvonen, Kivimäki, Pentti \& Vahtera, 2008) e, possivelmente, também contra o abuso de álcool, pois são menos presentes entre alcoolistas (Arthur, Hawkins, Pollard, Catalano \& Baglioni Jr, 2002). Também, apesar da controvérsia sobre a necessidade de treino em habilidades sociais no tratamento da esquizofrenia (Pilling et al., 2002), a inclusão dessa modalidade contribui para a prevenção de recaídas (Dixon et al., 2010; Halford \& Hayes, 1995).

No Brasil, Gomide, Salvo, Pinheiro e Sabbag (2005) mostraram que práticas educativas positivas estão relacionadas ao repertório de habilidades sociais dos familiares. Bandeira, Rocha, Freitas, Z.A.P. Del Prette e A. Del Prette (2006) verificaram que, quanto maior a escolaridade dos pais e seu nível socioeconômico, maior o escore das habilidades sociais; e que, quanto menor a importância atribuída pelos pais às habilidades sociais, menores os escores das crianças. Também Cia, Pamplin e Del Prette (2006) encontraram que os indicadores de envolvimento de pais e filhos correlacionam-se positivamente com o repertório de habilidades sociais das crianças; enquanto Bolsoni-Silva, Salina-Brandão, Versuti-Stoque e Rosin-Pinola (2008) constataram que a aquisição de habilidades sociais educativas promove melhoria quantitativa e qualitativa nas interações entre pais e filhos. Além disso, Bolsoni-Silva e Marturano (2010) indicaram que conflitos conjugais explicam parcialmente o surgimento de problemas de comportamento em pré-escolares, bem como A. Del Prette, Z.A.P. Del Prette, Gresham e Vance (2012) encontraram que características de comportamento social são fortes preditores de problemas de aprendizagem em crianças do ensino fundamental.

Tem-se observado no cenário nacional um interesse em mapear o comportamento dos universitários. Segundo Z.A.P. Del Prette e A. Del Prette (2001a), a preocupação com as habilidades sociais desse grupo justifica-se na medida em que se trata de um segmento populacional do qual tem sido exigida cada vez mais competência em administrar situações interpessoais. Nesse sentido, tanto Gerk e Cunha (2006) quanto Soares, Poubel e Mello (2009) encontraram correlações positivas entre habilidades sociais e adaptação ao ambiente acadêmico. As relações entre habilidades sociais e aceitação-rejeição entre universitários foi investigada por Bartholomeu, Carvalho, Silva, Miguel e Machado (2011), cujos resultados apontaram que a autoexposição a desconhecidos explica a aceitação para sair e estudar, entre as mulheres.

Também, as relações entre habilidades sociais e traços de personalidade foram investigadas por Bueno, S.M.S.S. Oliveira e J.C.S. Oliveira (2001), que encontraram que diferenças entre pessoas no que diz respeito ao enfrentamento com risco podem ser explicadas por extroversão e abertura para novas experiências. Diferenças em autoafirmação na expressão de afetos positivos são explicadas por socialização e equilíbrio emocional. Autoexposição a desconhecidos e a situações novas é relacionada com extroversão e equilíbrio emocional; autocontrole da agressividade é explicado pelo traço de personalidade denominado socialização. Conversação e desenvoltura social são relacionadas com o conjunto equilibrado de todos os cinco grandes fatores, exceto escrupulosidade. Numa pesquisa similar com universitários, Bartholomeu, Nunes e Machado (2008) encontraram correlações significativas entre amabilidade e autoafirmação, em todos os fatores do Inventário de Habilidades Sociais (IHS-Del-Prette), o que indica que efetivamente a personalidade está relacionada com diferentes aspectos da habilidade social.

Tendo em vista a relevância das habilidades sociais para o bom desempenho no ambiente universitário, e posteriormente, profissional, bem como sua associação com aspectos da personalidade e outras características psicológicas, este trabalho pretendeu identificar os padrões típicos de interações interpessoais de universitários e suas relações com as habilidades sociais. De modo particular, cogita-se que correlações entre medidas de habilidades sociais e posições interpessoais avaliadas pela versão brasileira do Checklist de Relações Interpessoais (Couto, Van Hattum, Vandenberghe \& Benfica, 2005) revelem uma fonte de evidência de validade para o Checklist. 


\section{Método}

\section{Participantes}

Participou desta pesquisa um grupo de 542 universitários de diversos cursos de duas instituições do sudeste de Goiás, com idade variando entre 18 e 55 anos $(M=21$ e $D P=5,48)$, sendo $64,4 \%$ dos sujeitos do sexo feminino (349). Na data de aplicação, 89,5\% dos sujeitos eram solteiros (485), 8,3\% casados (45), 1,3\% divorciados (7), 0,2\% viúvo (1), sendo que $0,7 \%$ não informaram o estado civil (4). Sobre a escolaridade, $4,4 \%$ já possuíam outro curso superior (24), enquanto 95,6\% o frequentavam pela primeira vez (254).

\section{Instrumentos}

Foram utilizados o Inventário de Habilidades Sociais (IHS-Del-Prette) e o Checklist de Relações Interpessoais - II (CLOIT-II).

O Inventário de Habilidades Sociais (IHS-DelPrette) foi desenvolvido para aferir o repertório de habilidades sociais usualmente requeridas em situações interpessoais cotidianas. A aferição é feita com base na estimativa que o respondente faz sobre a frequência com que reage da forma indicada em cada item, considerando o total de vezes em que se encontrou naquela situação. Foi testado e validado em vários estudos e disponibilizado por Z.A.P. Del Prette e A. Del Prette (2001b). O IHS-Del-Prette é composto por 38 itens, organizados em cinco fatores: Enfrentamento e autoafirmação com risco; Autoafirmação na expressão de afeto positivo; Conversação e desenvoltura social; Autoexposição a desconhecidos e situações novas; e Autocontrole da agressividade em situações aversivas (Z.A.P. Del Prette \& A. Del Prette, 2001b).

Por sua vez, o Checklist de Relações Interpessoais - II (CLOIT-II) é um inventário construído com a finalidade de mapear o comportamento interpessoal de pessoas-alvo. A forma de autoclassificação deve ser respondida pela pessoa-alvo, mediante 96 proposições (itens) que descrevem ações que podem ocorrer em interações entre pessoas. Todas as proposições são iniciadas com a expressão "Quando estou com outras pessoas... ", que fica no alto de cada página. Os participantes são solicitados a assinalar aqueles itens que condizem com seus comportamentos mais frequentes ao interagirem com outras pessoas. Alguns exemplos são: 1) ...tomo cuidado para não mostrar os meus sentimentos claramente; 2) ...sinto-me melhor deixando-as à vontade para fazer as coisas que elas querem; 3 ) ...demonstro sentir-me bem comigo mesmo;4) ...observo cuidadosamente para detectar reações que elas possam ter com o intuito de me prejudicar etc.

O inventário foi traduzido e adaptado por Couto et al. (2005) e teve suas propriedades psicométricas e aplicações para a população brasileira investigadas por Couto, Vandenberghe, Van Hattum e Campos (2006) e por Couto, Muniz, Vandenberghe e Van Hattum (2008). As proposições estão divididas em 16 escalas bidimensionais rotuladas pelas letras de $\mathrm{A}$ a $\mathrm{P}$ e distribuídas ao redor de um modelo circular em sentido anti-horário, a saber: Dominância (A), Competição (B), Desconfiança (C), Frieza Afetiva (D), Hostilidade (E), Isolamento (F), Inibição $(G)$, Insegurança (H), Submissão (I), Deferência $(\mathrm{J})$, Confiança (K), Calor Afetivo (L), "Amigabilidade" (M), Sociabilidade (N), Exposição (O), Segurança (P). Cada escala pode receber um escore bruto que varia entre 0 e 9 pontos. Os escores nas 16 escalas podem ser combinados com o objetivo de descrever padrões mais complexos de comportamentos. Por exemplo, os quadrantes são escalas combinadas pela soma dos resultados nas escalas que compõem cada quarto do círculo. As fórmulas são: Hostilidade-Dominância (Qdt-HD) $=0,707 \mathrm{~A}+0,924 \mathrm{~B}+\mathrm{C}+0,924 \mathrm{D}+0,707 \mathrm{E}$; Hostilidade-Submissão (Qdt-HS) $=0,707 \mathrm{E}+0,924 \mathrm{~F}+\mathrm{G}$ + 0,924 H + 0,707 I; "Amigabilidade"-Submissão (QdtAS) $=0,707 \mathrm{I}+0,924 \mathrm{~J}+\mathrm{K}+0,924 \mathrm{~L}+0,707 \mathrm{M} ;{ }^{\prime \prime}$ Amigabilidade"-Dominância (Qdt-AD) =0,707 M +0,924 N+ $\mathrm{O}+$ 0,924 P + 0,707 A. Também são computados escores separados para os quatro hemisférios possíveis do círculo.

As fórmulas para compor os escores dos hemisférios são: Dominância (Hmf-DOM) $=0,383 \mathrm{~N}+0,707 \mathrm{O}$ $+0,924 P+A+0,924 B+0,707 C+0,383$ D; Submissão $(\mathrm{Hmf}-\mathrm{SUB})=0,383 \mathrm{~F}+0,707 \mathrm{G}+0,924 \mathrm{H}+\mathrm{I}+0,924 \mathrm{~J}+$ $0,707 \mathrm{~K}+0,383 \mathrm{~L} ;$ "Amigabilidade" $(\mathrm{Hmf}-\mathrm{AMI})=0,383 \mathrm{~J}+$ $0,707 K+0,924 L+M+0,924 N+0,707 \mathrm{O}+0,383 \mathrm{P} ;$ Hostilidade $(\mathrm{Hmf}-\mathrm{HOS})=0,383 \mathrm{~B}+0,707 \mathrm{C}+0,924 \mathrm{D}+\mathrm{E}+$ $0,924 \mathrm{~F}+0,707 \mathrm{G}+0,383 \mathrm{H}$. Além disso, são providenciados escores que representam o vetor geral da pessoa-alvo em cada eixo do círculo, usando o peso trigono- 
métrico dos escores de um determinado protocolo. $\mathrm{O}$ escore no eixo vertical interpretado como Controle pode ser obtido pela fórmula: $\mathrm{CONTROLE}(\mathrm{EX}-\mathrm{COM})=\mathrm{A}$ $-\mathrm{I}+0,924(\mathrm{~B}+\mathrm{P}-\mathrm{H}-\mathrm{J})+0,707(\mathrm{C}+\mathrm{O}-\mathrm{G}-\mathrm{K})+0,383$ $(D+N-F-L)$, enquanto o escore no eixo horizontal interpretado como Filia pode ser obtido pela seguinte fórmula: FILIA (Ex-FIL) $=M-E+0,924(N+L-D-F)+$ 0,707 (O+K-C-G)+0,383 (P+J - B - H) (Kiesler, Goldston \&Schmidt, 1991).

\section{Procedimentos de coleta}

O projeto foi avaliado e autorizado pelo Comitê de Ética da Universidade Federal de Goiás, conforme parecer registrado sob protocolo no 214/2009, tendo todas as etapas da pesquisa sido conduzidas conforme os padrões exigidos pela Resolução 196/96 da Comissão Nacional de Ética em Pesquisa.

A coleta ocorreu de forma coletiva nas salas de aula e levou aproximadamente uma hora e trinta minutos. Os alunos foram convidados a participar da pesquisa e esclarecidos quanto aos procedimentos e ao caráter voluntário de sua participação. Aqueles que concordaram em participar assinaram o Termo de Consentimento Livre e Esclarecido e foram orientados a responder ao teste IHS-Del-Prette. Em seguida, eles foram solicitados a responder aos itens da forma de autoclassificação do CLOIT-II.

\section{Procedimentos de análise}

Ao final das aplicações, os testes foram corrigidos e os resultados dos protocolos foram tabulados em planilha eletrônica. Foram estimadas as estatísticas descritivas das respostas dos sujeitos ao IHS-Del-Prette e, em seguida, ao CLOIT-II.

Para atender aos objetivos da pesquisa, o escore total dos sujeitos no IHS-Del-Prette foi usado para separá-los em dois grupos, conforme sua habilidade social. O primeiro, composto por sujeitos que apresentaram escore geral de dois desvios-padrão abaixo da média do grupo ou menor, foi classificado como grupo de baixa habilidade social (Grupo 1). Já o segundo grupo foi composto por sujeitos que apresentaram valores de no mínimo dois desvios-padrão acima da média no escore geral e foi classificado com alta habilidade social
(Grupo 2). Em seguida, as posições interpessoais entre os dois grupos foram comparadas por meio da técnica estatística Análise de Variância (Anova) oneway, e verificadas as diferenças estatisticamente significativas. Ao final, foram estimados os coeficientes de correlação de Pearson ( $r$ ) entre as subescalas do IHS-Del-Prette e o perfil de relações interpessoais do CLOIT-II.

\section{Resultados e Discussão}

O padrão geral das habilidades sociais da amostra é representado na Tabela 1, onde se encontram as médias do escore total, dos escores fatoriais e dos percentis do grupo amostral, separado por gênero. Os sujeitos apresentaram padrões adequados de habilidades sociais, com percentis em torno da média em todas as dimensões. Os resultados não mostraram diferenças relevantes em relação ao gênero. Por outro lado, habilidades que envolvem Autoafirmação de afeto positivo são menos frequentes, tanto nos homens como nas mulheres, com pequena vantagem masculina. No entanto, os valores médios dos percentis nesse tipo de habilidade, apesar de caracterizar as maiores deficiências desse grupo, não são suficientemente baixas para indicação de intervenção.

O próximo passo foi estimar as características de relações interpessoais mais frequentes nos dois grupos e compará-las. Os resultados apontados na Figura 1 apresentam as médias de respostas por padrões de interações dos dois grupos. Pôde-se notar que, por um lado, os sujeitos do grupo de baixas habilidades sociais (Grupo 1) usam, para descrever suas interações interpessoais, mais características associadas ao hemisfério Submissão, mais especificamente ao quadrante Hostilidade-Submissão.

Ao se comparar o perfil interpessoal dos dois grupos, conforme observado na Figura 1, o Grupo 1 apresentou médias mais elevadas nas escalas do quadrante Hostilidade-Submissão, com diferenças significativas nas escalas Isolamento $[F(1,31)=10,29 ; p<0,00]$, Inibição $[F(1,31)=20,83 ; p<0,00]$, Insegurança $[F(1,31)=$ $6,39 ; p<0,02]$ e Submissão $[F(1,31)=17,37 ; p<0,00]$. Estes resultados indicam que pessoas com um repertório restrito em habilidades sociais tendem a assumir posições interpessoais mais tímidas ou caracterizadas por passividade, quando comparadas com pessoas com um reper- 
Tabela 1. Médias dos escores fatoriais e postos percentílicos (PP) do Inventário de Habilidade Social (IHS) por sexo. Catalão (GO), 2011.

\begin{tabular}{lccccc}
\hline \multirow{2}{*}{ Fatores do IHS } & \multicolumn{2}{c}{ Feminina } & & \multicolumn{2}{c}{ Masculina } \\
\cline { 2 - 3 } \cline { 5 - 6 } Enfrentamento/Autoafirmação com risco & Média & PP & & Média & PP \\
Autoafirmação na expressão de afeto positivo & 8,41 & $50 / 55$ & & 10,35 & 50 \\
Conversação e desenvoltura social & 8,03 & $20 / 25$ & & 7,51 & $25 / 30$ \\
Autoexposição a desconhecidos/situações novas & 7,23 & $65 / 70$ & & 7,30 & $65 / 70$ \\
Autocontrole da agressividade & 3,43 & $50 / 55$ & & 3,52 & $55 / 60$ \\
Escore Geral & 1,16 & 55 & & 1,38 & $45 / 50$ \\
\hline
\end{tabular}

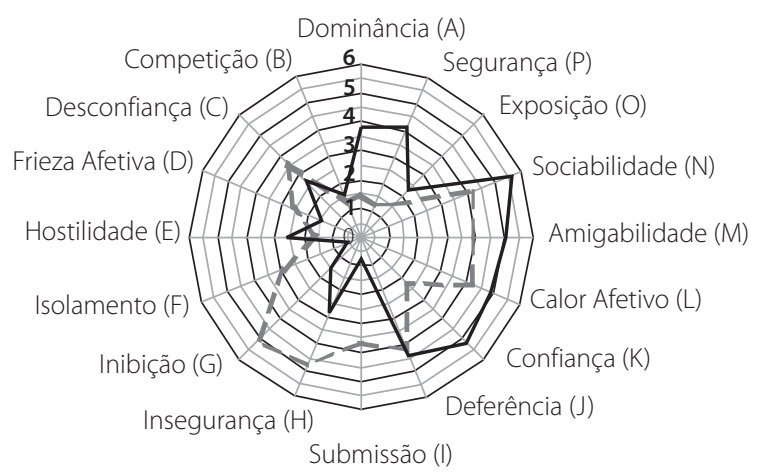

- - Baixa habilidades sociais $\quad$ - Alta habilidades sociais

Figura 1. Médias dos grupos nas posições interpessoais. Catalão (GO), 2011

tório mais amplo em habilidades sociais. Elas não se sentem confortáveis na companhia das pessoas, sentem dificuldade para se expressar, não se sentem capazes de realizar sozinhas as atividades, preferem não assumir responsabilidades e são facilmente persuadidas.

Já o Grupo 2 apresentou médias mais elevadas nas escalas do hemisfério "Amigabilidade", sendo significativas as diferenças nas escalas Confiança [F(1, $31)=26,15 ; p<0,00]$, Sociabilidade $[F(1,31)=3,21 ; p<0,08]$, Segurança $[F(1,216)=4,72 ; p<0,03]$ e Dominância $[F(1$, $216)=4,32 ; p<0,04]$. Os resultados reforçam a interpretação de que sujeitos com um repertório mais amplo de habilidades sociais assumem posições interpessoais baseadas em confiança, demonstram interesse no contato com as pessoas, demonstram sentir-se bem consigo mesmas e tendem a apresentar sugestões ou opiniões mais frequentemente do que aqueles com menos habilidades sociais.

A Tabela 2 apresenta as médias e os resultados 672 da Anova oneway, comparando os grupos nas escalas
Tabela 2. Médias e ANOVA entre os grupos nas escalas derivadas. Catalão (GO), 2011.

\begin{tabular}{lrrrr}
\hline $\begin{array}{l}\text { Escalas derivadas } \\
\text { do CLOIT-II }\end{array}$ & Alta HS & Baixa HS & $F$ & $p$ \\
\hline Hmf-DOM & 15,35 & 10,08 & 6,53 & 0,02 \\
Hmf-AMI & 23,28 & 16,45 & 7,07 & 0,01 \\
Hmf-SUB & 14,23 & 19,85 & 8,58 & 0,01 \\
Hmf-HOS & 9,15 & 15,13 & 5,43 & 0,03 \\
Qdt-AD & 17,61 & 10,43 & 16,39 & 0,00 \\
Qdt-AS & 17,75 & 15,33 & 1,53 & 0,23 \\
Qdt-HS & 7,03 & 15,88 & 18,92 & 0,00 \\
Qdt-HD & 10,03 & 9,34 & 0,96 & 0,76 \\
Eixo-Controle & 1,12 & $-9,77$ & 25,53 & 0,00 \\
Eixo-Filia & 14,13 & 1,32 & 14,95 & 0,00 \\
\hline
\end{tabular}

Hmf-DOM: Hemisfério Dominância; Hmf-AMI: Hemisfério Amigabilidade; Hmf-SUB: Hemisfério Submissão; Hmf-HOS: Hesmifério Hostilidade; Qdt-AD: Quadrante Amigabilidade-Dominância; Qdt-AS: Quadrante Amigabilidade-Submissão; Qdt-HS: Quadrante Hostilidade-Submissão; Qdt-HD: Quadrante Hostilidade Dominância; HS: Hostilidade-Submissão; CLOIT-II: Checklist de Interações Interpessoais - II.

derivadas. Conforme se pôde verificar, quando são examinados os eixos, hemisférios e quadrantes, o padrão geral das respostas mostra que o Grupo 1 evita posições de liderança, nas quais são necessárias a tomada de iniciativa e a aceitação de responsabilidades, assumindo mais frequentemente posições submissas, quando comparado com o Grupo 2.

Os dois grupos diferem mais especificamente em características relacionadas à submissão hostil, que é distinguida por posições interpessoais passivas mais ligadas às atitudes de isolamento e crenças de inadequação. Mas também diferem em "Amigabilidade" ativa, distinguida por posições ligadas ao prazer no contato social e as demonstrações de segurança. Esses resultados sugerem que pessoas com menos habilidades sociais assumem mais frequentemente posições in- 
terpessoais passivas, marcadas pela dificuldade em interagir apropriadamente.

O passo seguinte foi correlacionar os resultados relativos aos tipos de habilidades sociais e as posições interpessoais. Os resultados são apresentados nas Tabelas 3 e 4, que mostram os índices de correlação de Pearson. As correlações significativas variaram entre $r=-$ 0,42 e $r=0,35$ para as escalas principais (Tabela 3) e entre $r=-0,42$ e $r=0,38$ para as escalas derivadas (Tabela 4). Pôde-se notar que o número de correlações significativas e positivas é maior com as escalas do hemisfério "Amigabilidade", enquanto as correlações significativas e negativas são mais frequentes entre as escalas do quadrante Hostilidade-Submissão. Os coeficientes, de um modo geral, tendem a ser mais altos entre posições interpessoais que caracterizam determinados tipos de habilidades sociais. Por exemplo, as escalas Dominância e Segurança apresentam correlação positiva mais forte com a habilidade de Enfrentamento e Autoafirmação com risco, enquanto "Amigabilidade", Calor Afetivo e Confiança correlacionam-se com Autocontrole da agressividade em situações aversivas.

Esses resultados se assemelham aos encontrados por Bueno et al. (2001), que apontaram relações entre habilidade de Enfrentamento e traços de extroversão e abertura a novas experiências. De certa forma, posições interpessoais que podem ser atribuídas a pessoas extrovertidas e abertas, mesmo intuitivamente, são: disposição para participar ativamente das interações sociais, demonstrações de segurança nas próprias capacidades e também certa dose de confiança nas intenções alheias.

A relação de Autocontrole com socialização, também demonstrada no estudo de Bartholomeu et al. (2008), pode ser inferida nos resultados do presente estudo, por posições de cordialidade, demonstrações de carinho e compreensão, além de confiança. O mesmo padrão pôde ser observado nas correlações negativas: por exemplo, as escalas Frieza Afetiva, Isolamento, Inibição e Submissão apresentam correlações mais fortes com Autoafirmação na expressão de afeto positivo e, em seguida, com Conversação e desenvoltura social.

Quando são examinadas as escalas derivadas, verificou-se que as correlações mais altas são negativas com o hemisfério Hostilidade, especificamente com o quadrante Hostilidade-Submissão. Esse resultado sugere que, quanto mais frequentes as posições interpessoais hostis (e mais especificamente, hostis e submissas), menores as habilidades sociais (especialmente habilidades de expressar sentimento positivo e desenvoltura social, mas também enfrentamento, autoexposição a novidades e autocontrole da agressividade). Por outro lado, quanto mais características de dominância se

Tabela 3. Correlações entre interações interpessoais e habilidades sociais. Catalão (GO), 2011.

\begin{tabular}{|c|c|c|c|c|c|c|}
\hline \multirow[b]{2}{*}{ Escalas do CLOIT-II } & \multirow[b]{2}{*}{$\mathrm{EG}$} & \multirow[b]{2}{*}{ Enfrentamento } & \multicolumn{2}{|c|}{ Fatores do IHS } & \multirow[b]{2}{*}{ Autoexposição } & \multirow{2}{*}{$\begin{array}{l}\text { Autocontrole da } \\
\text { agressividade }\end{array}$} \\
\hline & & & $\begin{array}{c}\text { Expressão de } \\
\text { sentimento positivo }\end{array}$ & Desenvoltura social & & \\
\hline Dominância & $0,20^{* *}$ & $0,28^{* *}$ & $0,11^{*}$ & 0,03 & 0,08 & $-0,04$ \\
\hline Competição & 0,03 & $0,16^{* *}$ & 0,00 & $-0,06$ & 0,02 & $-0,12^{* *}$ \\
\hline Desconfiança & $-0,15^{* *}$ & 0,04 & $-0,20^{* *}$ & $-0,18^{* *}$ & $-0,12^{* *}$ & $-0,24^{* *}$ \\
\hline Frieza afetiva & $-0,22^{* *}$ & $-0,03$ & $-0,27^{* *}$ & $-0,19^{* *}$ & $-0,15^{* *}$ & $-0,16^{* *}$ \\
\hline Hostilidade & 0,04 & $0,12^{* *}$ & $-0,02$ & $-0,07$ & 0,06 & $-0,11^{*}$ \\
\hline Isolamento & $-0,36^{* *}$ & $-0,19^{* *}$ & $-0,36^{* *}$ & $-0,30^{* *}$ & $-0,18^{* *}$ & $-0,17^{* *}$ \\
\hline Inibição & $-0,36^{* *}$ & $-0,25^{* *}$ & $-0,32^{* *}$ & $-0,26^{* *}$ & $-0,20^{* *}$ & $-0,12^{* *}$ \\
\hline Insegurança & $-0,24^{* *}$ & $-0,16^{* *}$ & $-0,16^{* *}$ & $-0,24^{* *}$ & $-0,14^{* *}$ & $-0,14^{* *}$ \\
\hline Submissão & $-0,42^{* *}$ & $-0,30^{* *}$ & $-0,34^{* *}$ & $-0,31^{* *}$ & $-0,25^{* *}$ & $-0,12^{* *}$ \\
\hline Deferência & 0,08 & $0,09^{*}$ & $0,10^{*}$ & $-0,02$ & $0,11^{*}$ & $0,14^{* *}$ \\
\hline Confiança & $0,23^{* *}$ & $0,17^{* *}$ & $0,20^{* *}$ & $0,12^{* *}$ & $0,14^{* *}$ & $0,21^{* *}$ \\
\hline Calor afetivo & 0,07 & 0,03 & 0,07 & 0,03 & 0,06 & $0,24^{* *}$ \\
\hline "Amigabilidade" & $0,14^{* *}$ & 0,07 & $0,17^{* *}$ & 0,04 & $0,13^{* *}$ & $0,26^{* *}$ \\
\hline Sociabilidade & $0,10^{*}$ & 0,08 & 0,07 & 0,05 & $0,10^{*}$ & 0,07 \\
\hline Exposição & 0,02 & $0,14^{* *}$ & 0,08 & $-0,11^{*}$ & $-0,00$ & $-0,13^{* *}$ \\
\hline Segurança & $0,24^{* *}$ & $0,35^{* *}$ & $0,09^{*}$ & 0,10 & $0,13^{* *}$ & 0,05 \\
\hline
\end{tabular}

* Correlação é significativa no nível de $0.05 ;{ }^{* *}$ Correlação é significativa no nível de 0.01 .

EG: Escore Geral; IHS: Inventário de Habilidades Sociais. 
Tabela 4. Correlações entre as escalas derivadas e as habilidades sociais. Catalão (GO), 2011.

\begin{tabular}{|c|c|c|c|c|c|c|}
\hline \multirow{2}{*}{$\begin{array}{l}\text { Escalas derivadas } \\
\text { do CLOIT-II }\end{array}$} & \multirow{2}{*}{$\mathrm{EG}$} & \multirow{2}{*}{ Enfrentamento } & \multirow{2}{*}{$\begin{array}{c}\text { Expressão de } \\
\text { sentimento positivo }\end{array}$} & \multicolumn{2}{|c|}{ Fatores do IHS } & \multirow{2}{*}{$\begin{array}{l}\text { Autocontrole da } \\
\text { agressividade }\end{array}$} \\
\hline & & & & Desenvoltura social & Autoexposição & \\
\hline Qdt-HD & $-0,05$ & $0,15^{* *}$ & $-0,12^{* *}$ & $-0,14^{* *}$ & $-0,04$ & $-0,20^{* *}$ \\
\hline Qdt-HS & $-0,42^{* *}$ & $-0,25^{* *}$ & $-0,37^{* *}$ & $-0,36^{* *}$ & $-0,22^{* *}$ & $-0,20^{* *}$ \\
\hline Qdt-AS & $0,10^{*}$ & 0,06 & $0,11^{*}$ & $-0,01$ & $0,09^{*}$ & $0,24^{* *}$ \\
\hline Qdt-AD & $0,20^{* *}$ & $0,28^{* *}$ & $0,16^{* *}$ & 0,02 & $0,13^{* *}$ & 0,06 \\
\hline Hmf-DOM & $0,10^{*}$ & $0,26^{* *}$ & 0,02 & $-0,06$ & 0,04 & $-0,11^{* *}$ \\
\hline Hmf-SUB & $-0,23^{* *}$ & $-0,14^{* *}$ & $-0,17^{* *}$ & $-0,23^{* *}$ & $-0,11^{*}$ & 0,01 \\
\hline Hmf-AMl & $0,18^{* *}$ & $0,17^{* *}$ & $0,18^{* *}$ & 0,04 & $0,14^{* *}$ & $0,21^{* *}$ \\
\hline $\mathrm{Hmf}-\mathrm{HOS}$ & $-0,29^{* *}$ & $-0,08$ & $-0,32^{* *}$ & $-0,29^{* *}$ & $-0,16^{* *}$ & $-0,24^{* *}$ \\
\hline Eixo-Controle & $0,28^{* *}$ & $0,36^{* *}$ & $0,16^{* *}$ & $0,14^{* *}$ & $0,12^{* *}$ & $-0,11^{*}$ \\
\hline Eixo-Filia & $0,37^{* *}$ & $0,20^{* *}$ & $0,38^{* *}$ & $0,25^{* *}$ & $0,24^{* *}$ & $0,35^{* *}$ \\
\hline
\end{tabular}

${ }^{*}$ Correlação é significativa no nível de $0.05 ;{ }^{* *}$ Correlação é significativa no nível de 0.01 .

Qdt-HD: Quadrante Hostilidade-Dominância; Qdt-HS: Quadrante Hostilidade-Submissão; Qdt-AS: Quadrante Amigabilidade-Submissão; Hmf-DOM: Hemisfério Dominância; Hmf-SUB: Hemisfério Submissão; Hmf-AMI: Hemisfério Amigabilidade; Hmf-HOS: Hemisfério Hostilidade; IHS: Inventário de Habilidades Sociais; CLOIT-II: Checklist de Interações Interpessoais - II.

sobrepõem a características de submissão (especialmente dominância amigável), melhores são as habilidades de enfrentamento. E quanto mais características amigáveis se sobrepõem a características hostis, mais fortes estarão as habilidades de expressar sentimento positivo, desenvoltura social e controle da agressividade.

O padrão geral dos resultados sugere que posições interpessoais caracterizadas por hostilidade e submissão estão mais associadas a um repertório restrito de habilidades sociais que posições amigáveis e dominantes estão associadas a um repertório amplo de habilidades sociais. Isso sugere que pessoas com poucas habilidades sociais podem ser caracterizadas pela frequência de determinadas posições interpessoais, como isolamento, inibição e submissão, enquanto um repertório mais amplo em habilidades sociais não pode ser inferido simplesmente pela frequência de posições de sociabilidade, calor afetivo e deferência adotadas por uma pessoa.

Uma hipótese para explicar esses resultados é que um repertório extenso em habilidades sociais implica um padrão bastante diverso de posições interpessoais. Interações socialmente adequadas exigem necessariamente um certo grau de flexibilidade interpessoal, pela grande diversidade das circunstâncias sociais encontradas no cotidiano. Desse modo, os resultados indicam que pessoas com repertório mais amplo em

674 habilidades sociais podem ser caracterizadas pela variedade de posições interpessoais e, mais especificamente, pelo equilíbrio de posições de dominância e submissão e pela sobreposição de atitudes amigáveis sobre atitudes hostis (Tabelas 2 e 4).

Por outro lado, repertórios restritos em habilidades sociais associam-se mais especificamente a padrões restritos de características interpessoais. Esses resultados permitem a inferência de que um repertório mais amplo em habilidades sociais indica sujeitos socialmente mais competentes, especialmente quando avaliados pelos resultados em tarefas interpessoais, conforme os apontamentos de Dirks et al. (2007), Erickson e Shultz (2001) e Rose-Krasnor (1997).

\section{Considerações Finais}

O objetivo do presente estudo foi examinar as posições interpessoais típicas e suas relações com as habilidades sociais em universitários de um estado brasileiro. Ao proceder à análise dos resultados, pôde-se verificar que as posições interpessoais dos participantes variaram conforme seu perfil de habilidades sociais, demonstrando que pessoas com melhores habilidades sociais apresentam padrões de interação mais adequados. Por exemplo, é no grupo de altas habilidades que se notou a presença de maior variedade de padrões interpessoais e atitudes mais ativas, como a demonstração de interesse pelo ambiente social, busca pelo contato e crença na própria capacidade de contri- 
buir para as decisões sobre os caminhos que o grupo deve seguir. No grupo com habilidades sociais mais restritas, as pessoas apresentavam mais posições interpessoais passivas, como retraimento social, sentimento de inadequação, falta de interesse em assumir responsabilidades sociais etc.

Outro aspecto importante observado nos resultados foi que posições interpessoais específicas, socialmente indesejáveis, mostraram-se mais relacionadas com a falta de habilidade social, enquanto posições interpessoais desejáveis mostraram-se relacionadas com a presença dessas habilidades. Sugere-se que atitudes problemáticas específicas, quando aparecem na ciranda social, denunciam certa inépcia da pessoa nesse jogo inter-relacional, prejudicando sua competência social. Por outro lado, um desempenho socialmente hábil não é garantido apenas pela presença de determinadas posições interpessoais, por exemplo, a simples demonstração de cordialidade, deferência ou aceitação do outro. São necessários padrões mais complexos envolvendo a coordenação de posições interpessoais. A pessoa deve mesclar tipos de atitudes amigáveis, ora ativas, ora passivas, e principalmente, sobrepor as atitudes amigáveis às hostis, conforme pôde ser encontrado na literatura especializada e também ilustrado pelos resultados aqui relatados.

De forma particular, os resultados encontrados podem ser considerados como fonte de evidência de validade para CLOIT-II, uma vez que as variáveis interpessoais medidas por ele encontram relação com variáveis com as quais teoricamente deveriam se relacionar. Era esperado que padrões de interação interpessoal negativos apresentassem correlações negativas com os indicadores de habilidades sociais, assim como que posições interpessoais positivas apresentassem correlações positivas com esses indicadores. Além disso, a compreensão das dinâmicas interpessoais foi reforçada pela observação de que as correlações negativas são mais fortes que as positivas, como discutido no parágrafo anterior.

Um apontamento a ser feito com relação aos resultados do presente estudo é que o meio universitário é um ambiente complexo, pois as pessoas estão à mercê de diversas exigências sociais. Por um lado, o fato de frequentarem a universidade e de serem avaliadas diuturnamente do ponto de vista acadêmico, sugere a forte exigência de retraimento social para realizar as demandas do estudo. Por outro lado, o mesmo ambiente dispõe de vários reforçadores para o comportamento exploratório, pois reúne pessoas de diferentes faixas etárias, regiões e culturas, além de apresentar forte apelo para a interação social. Dessa forma, tais características podem ter contribuído para um aumento da influência de desejabilidade social, elemento comumente conhecido nos resultados de instrumentos de autorrelato, nas respostas dadas aos itens das escalas modificando a intensidade e forma das expectativas interpessoais e também de habilidades sociais declaradas.

De todo modo, tomadas as devidas precauções, os resultados podem ser considerados descrições razoáveis dos fenômenos estudados. Aconselha-se que novos estudos sejam desenvolvidos na tentativa de replicar esses resultados, com a utilização de métodos diferentes que permitam triangulação para melhorar as observações sobre as dimensões psicológicas estudadas.

\section{Referências}

Abroms, L., Simons-Morton, B., Haynie, D. L., \& Chen, R. (2005). Psychosocial predictors of smoking trajectories during middle and high school. Addiction, 100 (6), 852-861.

Argyle, M., \& Lu, L. (1990). Happiness and social skills. Personality and Individual Differences, 11 (12), 1255-1261.

Arthur, M. W., Hawkins, J. D., Pollard, J. A., Catalano, R. E., \& Baglioni Jr., A. J. (2002). Measuring risk and protective factors for substance use, delinquency, and other adolescent problem behaviors. Evaluation Review, 26 (6), 575-601.

Bandeira, M., Rocha, S. S., Freitas, L. C., Del Prette, Z. A. P., \& Del Prette, A. (2006). Habilidades sociais e variáveis sociodemográficas em estudantes do ensino fundamental. Psicologia em Estudo, 3, 541-549.

Bartholomeu, D., Carvalho, L. F., Silva, M. C. R., Miguel, F. K., \& Machado, A. A. (2011). Aceitação e rejeição entre pares e habilidades sociais em universitários. Estudos de Psicologia (Natal), 16 (2), 155-162.

Bartholomeu, D., Nunes, C. H. S. S., \& Machado, A. A. (2008). Traços de personalidade e habilidades sociais em universitários. Psico-USF, 13 (1), 41-50.

Bellack, A. S., \& Morrison, R. L. (1982). Interpersonal dysfunction. In A. S. Bellack, M. Hersen \& A. E. Kazdin. International handbook of behavior modification and therapy. New York: Plenum.

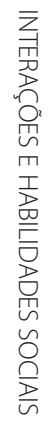 \\ 675}


Berscheid, E., \& Peplau, L. A. (1983). The emerging science of relationship. In H. H. Kelley, E. Berscheid, A. Christensen, J. H. Harvey, T. L. Huston, G. Levinger, et. al. (Orgs.), Close relationships. San Francisco: Freeman and Company.

Bolsoni-Silva, A. T., \&Marturano, E. M. (2010). Relacionamento conjugal, problemas de comportamento e habilidades sociais em pré-escolares. Psicologia: Teoria e Pesquisa, 26 (1), 67-75.

Bolsoni-Silva, A. T., Salina-Brandrão, A., Versuti-Stoque, F. M., \& Rosin-Pinola, A. R. (2008). Avaliação de um programa de intervenção de habilidades sociais educativas parentais: um estudo-piloto. Psicologia: Ciência e Profissão, 28 (1), 18-33.

Bueno, J. M. H., Oliveira, S. M. S. S., \& Oliveira, J. C. S. (2001). Um estudo correlacional entre habilidades sociais e traços de personalidade. PsicoUSF, 6 (1), 31-38.

Cavell , T. A., Meehan, B. T., \& Fiala, S. E. (2003). Assessing social competence in children and adolescents. In C. R. Reynolds \& R. W. Kamphaus (Eds.), Handbook of psychological and educational assessment of children: personality, behavior, and context ( $2^{\text {nd }}$ ed., pp.433-454). New York: Guilford Press.

Cia, F., Pamplin, R. C. O., \& Del Prette, Z. A. P. (2006). Comunicação e participação pais-filhos: correlação com habilidades sociais e problemas de comportamento dos filhos. Paidéia, 16 (35), 395-406.

Couto, G., Muniz, M., Vandenberghe, L. M. A., \& Van Hattum, A. C. (2008). Diferenças relacionadas ao sexo observadas no Checklist de Relações Interpessoais: revisado. Avaliação Psicológica, 7 (3), 347-357.

Couto, G., Van Hattum. A. C. F. M., Vandenberghe, L. M. A., \& Benfica, E. (2005). Tradução, análise semântica e adaptação do Check List of Interpersonal Transactions: revisado. Avaliação Psicológica, 4 (1), 45-57.

Couto, G., Vandenberghe. L., Van Hattum, A. C. F. M., \& Campos, H. R. (2006). Propriedades psicométricas do checklist de relações interpessoais: revisado. Psicologia Argumento, 24 (47), 15-28.

Del Prette, Z. A. P., \& Del Prette, A. (1999). Psicologia das habilidades sociais: terapia e educação. Petrópolis: Vozes.

Del Prette, Z. A. P., \& Del Prette, A. (2001a). Psicologia das relações interpessoais: vivências para o trabalho em grupo. Petrópolis: Vozes.

Del Prette, Z. A. P., \& Del Prette, A. (2001b). Manual de aplicação, apuração e interpretação do Inventário de Habilidades Sociais (IHS-Del-Prette). São Paulo: Casa do Psicólogo.

Del Prette, A., Del Prette, Z. A. P. Gresham, F. M., \& Vance, M. J. (2012). Role of social performance in predicting learning problems: prediction of risk using logistic regression analysis. School Psychology Journal, 2, 1-16.

Dirks, M. A., Treat, T. A., \& Robin-Weersing, V. (2007). Integrating theoretical, measurement, and intervention models of youth social competence. Clinical Psychology Review, 27 (3), 327-347.
Dixon, L. B., Dickerson, F., Bellack, A. S., Bennett, M., Dickerson, D., Goldberg, R. W., et al. (2010). The 2009 schizophrenia PORT psychosocial treatment recommendations and summary statements. Schizophrenia Bulletin, 36 (1), 48-70.

Erickson, F., \& Shultz, J. (2001). When is a context? some issues and methods in the analysis of social competence. In M. Cole, Y. Engeström \& O. Vasquez (Eds.), Mind, culture, and activity: seminal papers from the laboratory of Comparative Human Cognition. Cambridge: Cambridge University Press.

Garmezy, N., \& Masten, A. (1994). Chronic adversities. In M. Rutter, E. Taylor \& L. Herson (Orgs.), Child and adolescent psychiatry (pp.191-207). Oxford: Blackwell Scientific Publication.

Gerk, E., \& Cunha, S. M. (2006). As habilidades sociais na adaptação de estudantes ao ensino superior. In M. Bandeira, Z. A. P. Del Prette \& A. Del Prette (Orgs.), Estudos sobre habilidades sociais e relacionamento interpessoal (pp.181-198). São Paulo: Casa do Psicólogo.

Gomide, P. I. C., Salvo, C. G., Pinheiro, D. P. N., \& Sabbag, G. M. (2005). Correlação entre práticas educativas, depressão, stress e habilidades sociais. Psico-USF, 10 (2), 169-178.

Halford, W. K., \& Hayes, R. L. (1995). Social Skill in Schizophrenia: assessing in relationship Between social skills, psychopathology and community functioning. Social Psychiatry and Psychiatric Epidemiology, 30 (1), 14-19.

Kapp-Simon, K. A., McGuire, D. E., Long, B. C., \& Simon, D. J. (2005). Addressing quality of life issues in adolescents: social skills interventions. Cleft Palate and Craniofacial Journal, 42 (1), 45-50.

Kiesler, D. J., Goldston, C. S., \& Schmidt, J. A. (1991). Manual for check list of interpersonal transactions: revised and check list of psychotherapy transactions - revised. Virginia: Virginia Commonwealth University.

Levinger, G. (1983). Development and change. In H. H. Kelley, E. Berscheid, A. Christensen, J. H. Havey, T. L. Histon, G. Levinger, et al. (Orgs.), Close relationships (pp.315-359). New York: Freeman and Company.

Lillvist, A., Sandberg, A., Björck-Äkesson, E., \& Granlund, M. (2010). The construct of social competence-how preschool teachers define social competence in young children. International Journal of Early Childhood, 41 (1), 51-68.

Luthar, S. S., Cicchetti, D., \& Becker, B. (2000). The Construct of Resilience: a critical evaluation and guidelines for future work. Child Development, 71 (3), 543-562.

Pilling, S., Bebbington, P., Kuipers, E., Garety, P., Geddes, J., Martindale, B., et al. (2002). Psychological treatments in schizophrenia: Il. Meta-analyses of randomized controlled trials of social skills training and cognitive remediation. Psychological Medicine, 32 (5), 783-791.

Rose-Krasnor, L. (1997). The nature of social competence: a theoretical review. Social Development, 6 (1), 111-136.

Rusbult, C. E., \& Buunk, B. P. (1993). Commitment processes in close relationships: an interdependence analysis. Journal of Social and Personal Relationship, 10 (2), 175-204. 
Sardinha, A., Falcone, E. M. O., \& Ferreira, M. C. (2009). As relações entre a satisfação conjugal e as habilidades sociais percebidas no cônjuge. Psicologia: Teoria e Pesquisa, 25 (3), 395-402.

Soares, A. B., Poubel, L. M., \& Mello, T. V. S. (2009). Habilidades sociais e adaptação acadêmica: um estudo comparativo em instituições de ensino público e privado. Aletheia, 30, 27-42.

Sousa, L., Galante, H., \& Figueiredo, D. (2003). Qualidade de vida e bem-estar dos idosos: um estudo exploratório na população portuguesa. Revista de Saúde Pública, 37 (3), 364-371.

Stanley, S. M., Amato, P. R., Johnson, C. A., \& Markman, H. J. (2006). Premarital education, marital quality, and marital stability: findings from a large, random household survey. Journal of Family Psychology, 20 (1), 117-126.

Sullivan, H. S. (1953). The interpersonal theory of psychiatry. New York: Norton.

Väänänen, A., Kouvonen, A., Kivimäki, M., Pentti, J., \& Vahtera, J. (2008) Social support, network heterogeneity, and smoking behavior in women: the 10 - Town Study. American Journal of Health Promotion, 22 (4), 246-252.

Recebido em: 16/2/2011

Versão final em: 23/4/2012

Aprovado em: 18/5/2012 
